\title{
Hepatobiliary fascioliasis treated at endoscopic retrograde cholangiopancreatography
}

In most parts of Asia, hepatobiliary fascioliasis is sporadic and has been traditionally attributed to travel to endemic zones. Recently the World Health Organization has described fascioliasis as an 'emerging human disease', with cases being increasingly reported from non-endemic zones. A 28-year-old woman presented with a 2-month history of abdominal pain. Examination revealed fever and tender hepatomegaly. Laboratory investigation showed the following results: hemoglobin, $9.4 \mathrm{~g} / \mathrm{dL}$; white cell count, 9100 cells $/ \mathrm{mm}^{3}$ (24\% eosinophils); aspartate aminotransferase, $64 \mathrm{IU} / \mathrm{L}$; alanine aminotransferase, $84 \mathrm{IU} / \mathrm{L}$; and alkaline phosphatase, 746 IU/L. Computed tomography (CT) scan demonstrated multiple complex hepatic cysts ( $\bullet$ Fig. 1 ).

At endoscopic retrograde cholangiopancreatography (ERCP) the cholangiogram showed a non-dilated common bile duct with multiple irregular ill-defined filling defects. During balloon extraction after sphincterotomy ( Video 1), live flat leafshaped adult Fasciola hepatica were withdrawn from the bile duct ( $\bullet$ Fig. 2 ).

The patient was treated with triclabendazole $(10 \mathrm{mg} / \mathrm{kg})$ in two divided doses, and continues to be symptom-free after 6 months of follow-up.

Human hepatobiliary infection by this trematode has two distinct phases: an acute hepatic phase and a chronic biliary phase. The acute phase is clinically manifested by upper abdominal pain, fever, hepatomegaly, and intense eosinophilia. In the chronic biliary phase, the adult worms attach to the mucosal lining of the biliary tree, where they may survive for years, causing periodic biliary obstruction.

ERCP in biliary fascioliasis may be normal in early disease and closely mimics primary sclerosing cholangitis in the chronic phase [1]. A short sphincterotomy is often adequate to achieve removal of these soft parasites [2]. Some authors have described intraductal endoscopic treatment of massive biliary fascioliasis by means of $10 \%$ povidone iodine solution $[3,4]$. Although results have been encouraging in small groups of patients, larger studies need to validate the safety of intraductal endoscopic treatment before accepting it as a standard endoscopic intervention for biliary fascioliasis.

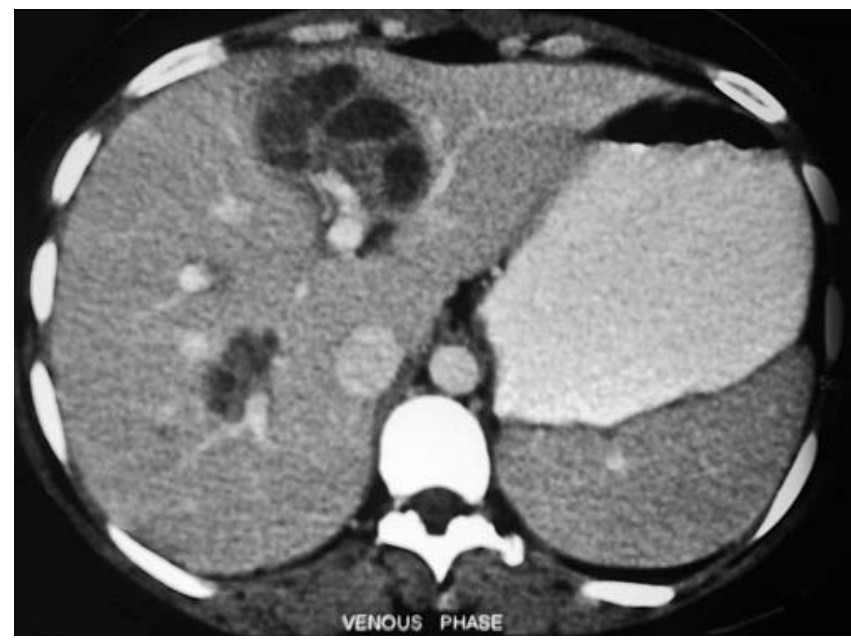

Fig. 1 Portal venous phase computed tomography scan showing multiple hypodense non-enhancing complex cystic lesions in the liver.

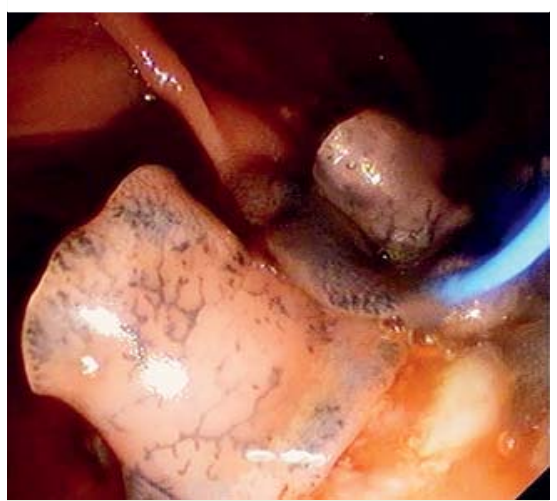

Fig. 2 Endoscopic image of a live Fasciola hepatica emerging from the bile duct during balloon extraction at endoscopic retrograde cholangiopancreatography.

In view of the global increase of migrant populations and international food trade, boundaries between endemic and nonendemic zones for human fascioliasis are being blurred. Hence, diagnosis of human fascioliasis should be strongly suspected in patients presenting with the triad of abdominal pain, hepatomegaly, and hypodense liver parenchymal lesion with or without peripheral eosinophilia even in non-endemic regions.

\section{Competing interests: None}

Endoscopy_UCTN_Code_CCL_1AZ_2AN

\section{Video 1}

Endoscopic sphincterotomy and balloon extraction of live Fasciola hepatica from the bile duct.
M. K. Goenka ${ }^{1}$, S. Majumder ${ }^{1}$,

P. K. Sethy ${ }^{1}$, S. Kumar ${ }^{1}$, U. Goenka ${ }^{2}$ Institute of Gastroenterology, Apollo Gleneagles Hospital, Kolkata, India

2 Department of Radiology, Apollo Gleneagles Hospital, Kolkata, India

\section{References}

1 Hauser SC, Bynum TE. Abnormalities on ERCP in a case of human fascioliasis. Gastrointest Endosc 1984; 30: 80-82

2 Dias LM, Suva R, Viana HL et al. Biliary fascioliasis: diagnosis, treatment and followup by ERCP. Gastrointest Endosc 1996; 43: $616-620$

3 Dowidar N, El Sayad M, Osman M et al. Endoscopic therapy of fascioliasis resistant to oral therapy. Gastrointest Endosc 1999; 50: $345-351$

4 Roig GV. Hepatic fascioliasis in the Americas: a new challenge for therapeutic endoscopy. Gastrointest Endosc 2002; 56: 315-317

Bibliography

Dol $10.1055 / \mathrm{s}-0029-1243907$

Endoscopy 2010; 42: E103

(c) Georg Thieme Verlag KG Stuttgart · New York . ISSN 0013-726X

\section{Corresponding author}

M. K. Goenka, MD

Institute of Gastroenterology,

Apollo Gleneagles Hospital

$\mathrm{BH}-97$, Sector 2

Salt Lake City

Calcutta 700091

India

Fax: $+91-33-23205218$

dr_mkgoenka@yahoo.co.in 